\title{
Sorafenib treatment for recurrent stage TI bilateral renal cell carcinoma in patients with Von Hippel- Lindau disease: A case report and literature review
}

\author{
Kyung Hwa Choi, MD; Young Dong Yu, MD; Moon Hyung Kang, MD; Dong Soo Park, MD
}

Department of Urology, CHA Bundang Medical Center, CHA University, Seongnam, Korea

Cite as: Can Urol Assoc J 2015;(9-10):E651-3. http://dx.doi.org/10.5489/cuaj.2863 Published online September 9, 2015.

\section{Abstract}

Renal cell carcinoma (RCC) with Von Hippel-Lindau (VHL) syndrome is associated with multiple recurrences and a young age at diagnosis. Therefore the primary goal of treatment is to stabilize the disease, minimizing the surgical resection and preserving the renal function in the patients with VHL who have developing RCC nodules after initial treatment. This is the first case report of $\mathrm{VHL}$ disease, with long-term stable disease, treated with a half dose of sorafenib after surgical resection and radiofrequency ablation for multiple recurrent stage T1 masses. We discuss the efficacy and safety of low-dose sorafenib treatment and review RCC in a patient with VHL disease.

\section{Introduction}

Because renal cell carcinoma (RCC) with Von Hippel-Lindau (VHL) syndrome is associated with multiple recurrences and a young age at diagnosis, it usually needs repeated intervention. The therapeutic goal is oncologic control, while preserving renal function. We present unique cases of multiple recurrent stage T1a VHL-RCC, which were well-stabilized with sorafenib treatment.

\section{Case 1}

A 38-year-old woman visited our clinic for recurrent RCC. She was genetically diagnosed with VHL disease 10 years prior at another hospital, and underwent right partial nephrectomy for RCC and surgical resection of hemangioblastoma in the cerebellum at that time. She also had retinal hemangioblastoma and C2-4 spinal cord hemangioblastoma. Her father and her son were also diagnosed with VHL disease. The abdominal computed tomography (CT) at the other hospital revealed multiple RCCs in both kidneys (largest $5.2 \mathrm{~cm}$ ) and multiple pancreatic cysts (Fig. 1, part A). There was no evidence of distant metastasis.

For the multiple RCC, we performed radiofrequency ablation (RFA) for the largest RCC $(5.2 \mathrm{~cm})$, and bilateral partial nephrectomies for right 5 RCCs (largest one $2 \mathrm{~cm}$ ) and left 5 RCCs (largest one $3.5 \mathrm{~cm}$ ). The pathologic diagnosis was Fuhrman nuclear grade (FG) 1, stage T1 clear cell RCC for all tumours. At discharge, the patient's serum creatinine level was normalized (preoperative $1.1 \mathrm{mg} / \mathrm{dL}$, postoperative $>1.1 \mathrm{mg} / \mathrm{dL}$ ). Three months after surgery, the patient started on a half-dose sorafenib (400 $\mathrm{mg}$, daily) for the remaining bilateral RCCs (right: 3.4, 3, 2.5, $2 \mathrm{~cm}$ size, left: $<1 \mathrm{~cm}$ size, multiple) (Fig. 1, part B). We used the half-dose sorafenib treatment because (1) the full dose (800 mg, daily) frequently causes severe hand-foot-skin problems and other adverse effects (i.e., diarrhea) especially in initial 3 to 4 months; (2) the Fuhrman nuclear grade 1 RCC could be controlled with the half dose; (3) the patient had a low body weight (50 kg); and (4) we assumed that sunitinib was not suitable for patients scheduled for long-term treatment due to its severe adverse effects, such as hematologic complication compared to sorafenib. Six months after sorafenib administration, the main RCCs, including the largest one, regressed and reached partial response according to the Response Evaluation Criteria in Solid Tumors (RECIST) criteria. (Fig. 1, part C). At 9 months, the patient complained of diarrhea (Common Terminology Criteria for Adverse Events [CTCAE] grade 1); however, she tolerably received sorafenib maintenance with antidiarrheal treatment due to intermittent diarrhea lasting less than 6 months. The size of the pancreatic cyst slightly decreased (from 6.3 to $>5.9 \mathrm{~cm}$ ) during the treatment periods, but other lesions showed no change. She is still responding to half-dose sorafenib treatment at 65 months (Fig. 1, part D).

\section{Case 2}

A 37-year-old man was referred to our urologic department for RCC, found during a regular check-up. The CT revealed a 


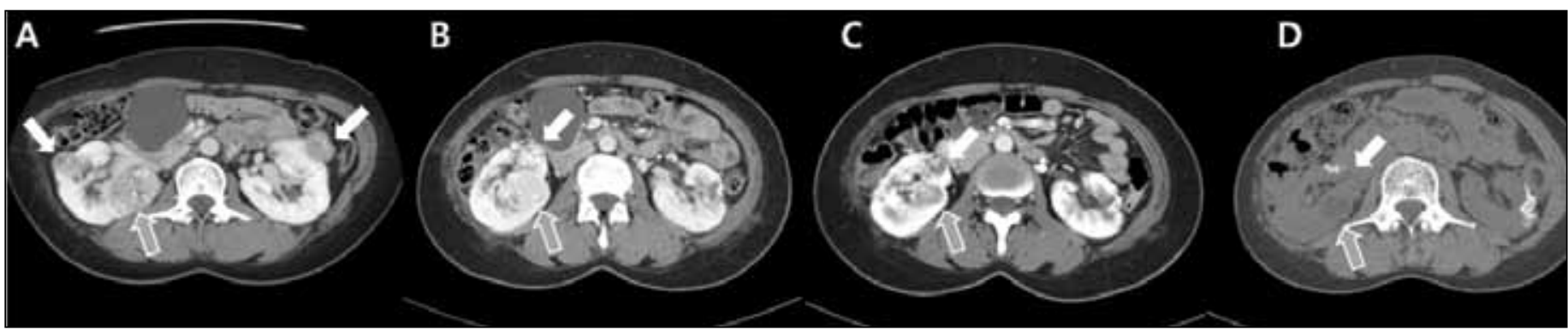

Fig. 1. Response of $\mathrm{T} 1$ stage Von Hippel-Lindau-renal cell carcinoma (RCC) to sorafenib (Case 1). A: Pre-treatment computed tomography image (white arrow multiple RCC treated by partial nephrectomy, gray arrow largest RCC which treated by radiofrequency ablation [RFA]). B: Sorafenib start on for remaining multiple RCC (white arrow) 3 month after previous partial nephrectomy and RFA. C: Partial response (white arrow) after 6 month sorafenib treatment (gray arrow; RCC which treated by RFA). D: Recent follow up non-contrast computed tomography scan (at 65 months).

5-cm sized RCC with multiple renal cysts and multiple small pancreatic cysts (Fig. 2, part A). He had brain surgery for a benign cerebellar tumour 22 years prior, but he had neither family history of brain cancer nor VHL. In VHL mutation analysis, VHL-exon deletion was suspected. Partial nephrectomy was applied for the 5-cm size clear cell type RCC. After 6 months, another cystic RCC was suspected at the other side of kidney, and also treated with partial nephrectomy. Serum creatinine level was stabilized at slightly higher level than normal range $(1.3 \mathrm{mg} / \mathrm{dL})$ after surgeries. During the secondary postoperative period, many $(\mathrm{n}=6-49) 1$ - to $2.4-\mathrm{cm}$ size multiple RCCs recurred under watchful waiting (Fig. 2, part B). At 55 months, the disease progressed $(2.4->3.2 \mathrm{~cm}$ ) (Fig. 2 , part C) and the patient started taking $800 \mathrm{mg}$ of sorafenib daily. However, he needed a dose reduction $(400 \mathrm{mg}$, daily) 3 months later due to CTCAE grade 2 diarrhea; he tolerated treatment after the dose adjustment. The disease reached a partial response $(3.2->2.2 \mathrm{~cm})$ at 2 months after sorafenib administration (Fig. 2, part D) and the patient still responded to treatment at the recent follow-up (96 months after his first surgery, at 35 months after sorafenib), revealed via a noncontrast CT (Fig. 2, part E).

\section{Discussion}

To the best of our knowledge, this is the first report showing that sorafenib could effectively control the recurrent of stage T1 VHL-RCC.
$\mathrm{VHL}$ disease is the most well-known syndrome, which associated with the hereditary RCC disease. VHL gene mutation results in the autosomal dominant disorder ${ }^{1}$ and characterized by various diseases. Among them, VHL Phenotype 1 and $2 \mathrm{~B}$, which are associated with retinal hemangioblastoma, hemangioblastoma of the brain or spinal cord, pancreatic cyst and neuroendocrine tumor, related to high-risk RCC. $^{2}$

VHL-RCC present multiple, recurrent features and diagnosed at a young age, but only $25 \%$ are known to have metastatic RCC. ${ }^{3}$ Therefore, treatment modality is determined according to tumour size, and the number and growth rate to preserve renal function. ${ }^{2}$ For stage T1 VHL-RCC, nephronsparing surgery and focal therapy, such as cryoablation and RFA for large or multiple RCC, as well as watchful waiting for masses less than 3 to $4 \mathrm{~cm}$, are considered standard therapy. ${ }^{4,5}$ However, in surgical resection, the recurrence rate is relatively high $(50 \%)$ and $25 \%$ of cases need reoperation; ${ }^{6}$ surgical resection or RFA, however, cannot be applied repetitively due to the nephron loss and possible complications. ${ }^{7}$ Postsurgical dialysis or renal transplantation, the final treatment options, also have been used for non-metastatic VHL-RCC even in young age. ${ }^{8}$

Therefore in this study, we applied a medical treatment, which is used for metastatic RCC to preserve the normal renal parenchyme. Sorafenib, tyrosine kinase inhibitor $(\mathrm{TKI})$, is the first-line treatment for metastatic clear cell RCC and targets the vascular endothelial growth factor receptor

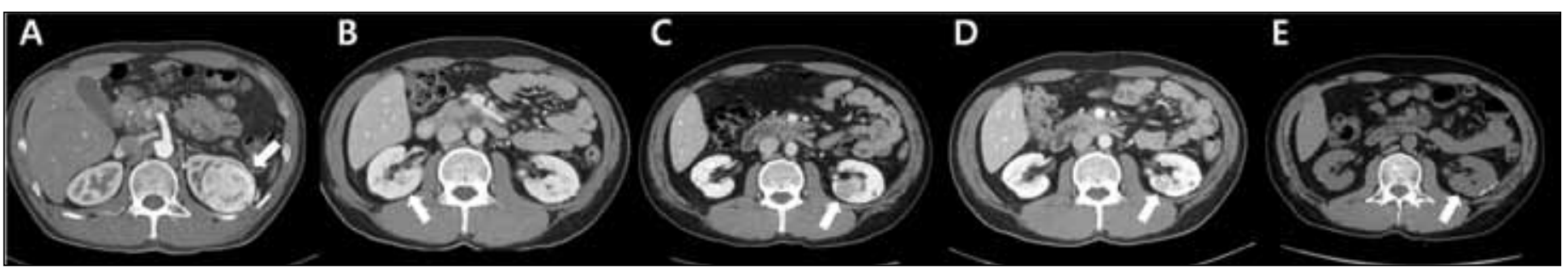

Fig. 2. Response of T1 stage VHL-RCC to sorafenib (Case 1). A.: Pre-treatment computed tomography (CT) image (white arrow; large RCC), B: 1-cm size right RCC recurred after the second partial nephrectomy (white arrow). C: Sorafenib administration started for the recurred RCC (white arrow) 55 months post-operation. D: Partial response (white arrow) after 2 months of sorafenib treatment. E: Non-contrast CT scan from the recent follow up (at 35 months after sorafenib, white arrow). 
(VEGFR) and other receptors affected by VHL-HIF cancer pathway activation. ${ }^{9}$ However, sorafenib for non-metastatic VHL-RCC has not been reported. Sunitinib is another TKI, which is the first-line treatment for metastatic RCC; it has been reported in non-metastatic VHL-RCC twice. Jonasch and colleagues reported the sunitinib treatment outcome for 1 - to $3-\mathrm{cm}$ sized VHL-RCC in $15 \mathrm{VHL}$ patients. At 48 weeks after $50 \mathrm{mg}$ full-dose sunitinib treatment (4 weeks' treatment followed by 2 weeks' rest), 6 of the 18 (33\%) lesions showed partial response, 10 (56\%) showed stable disease and $2(11 \%)$ showed progressive disease. However, dose reductions (to $25 \mathrm{mg}$ from $37.5 \mathrm{mg}$ ) were needed in 10 of the $15(67 \%)$ patients because of grade 2 to 3 toxicity. ${ }^{10}$ Roma and colleagues also reported the first-line sunitinib in VHL-RCC patients with recurrence or progression of multifocal kidney lesion $(n=4)$ and/or distant metastasis $(n=10)$. In their study, $64.3 \%$ of patients achieved partial response and others achieved stable disease at the maximum response period. However, 6 patients progressed and 3 patients died at a median follow-up of 37 months. The patients with toxicity grade $\geq 2$ also needed a dose reduction from $50 \mathrm{mg}$ to 25 mg. ${ }^{11}$

Half-dose sorafenib is comparable to sunitinib in oncologic outcome and long-term stability, with relatively little complication.

\section{Conclusion}

The low-dose sorafenib maintenance could be a tolerable and effective long-term treatment option for recurrent stage T1 VHL-RCC patients, who need maximal preservation of renal function.
Competing interests: The authors all declare no competing financial or personal interests.

This paper has been peer-reviewed.

\section{References}

1. Latif $F$, Tory K, Gnarra J, et al. Identification of the von Hippel-Lindau disease tumor suppressor gene. Science 1993;260:1317-20. http://dx.doi.org/10.1126/science.8493574

2. Bausch $B$, Jilg $C$, Glasker $S$, et al. Renal cancer in von Hippel-Lindau disease and related syndromes. Nat Rev Nephrol 2013;9:529-38. http://dx.doi.org/10.1038/nrneph.2013.144

3. Friedrich CA. Von Hippel-Lindau syndrome: A pleomorphic condition. Cancer 1999:86 (11 Suppl):2478-82.

4. Matin SF, Ahrar K, Wood CG, et al. Patterns of intervention for renal lesions in von Hippe-lindau disease. BJU Int 2008;102:940-5. http://dx.doi.org/10.1111/i.1464-410X.2008.07718.x

5. Gupta GN, Peterson J, Thakore KN, et al. Oncological outcomes of partial nephrectomy for multifocal renal cell carcinoma greater than $4 \mathrm{~cm}$. J Urol 2010;184:59-63. http://dx.doi.org/10.1016/j. juro.2010.03.035

6. Ploussard $G$, Droupy $S$, Ferlicot $S$, et al. Local recurrence after nephron-sparing surgery in von Hippel-Lindau disease. Urology 2007;70:435-9. http://dx.doi.org/10.1016/i.urology.2007.04.040

7. Weizer AZ, Raj GV. Complications after percutaneous radiofrequency ablation of renal tumors. Urology 2005;66:1176-80.

8. Steinbach F, Novick AC, Shoskes D. Renal transplantation in patients with renal cell carcinoma and von Hippe-Lindau disease. Urology 1994;44:760-3. http://dx.doi.org/10.1016/S0090-4295(94)80224-6

9. Randall JM, Millard F, Kuzzrock R. Molecular aberrations, targeted therapy, and renal cell carcinoma: Current state-of-the-art. Cancer and Metastasis Rev 2014;33:1 109-24. http://dx.doi.org/10.1007/ s10555-014-9533-1

10. Jonasch E, McCutcheon IE, Waguespack SG, et al. Pilot trial of sunitinib therapy in patients with von Hippe-Lindau disease. Ann Oncol 2011;22:2661-6. http://dx.doi.org/10.1093/annonc/mdr011

11. Roma A, Maruzzo M, Basso U, et al. First-Line sunitinib in patients with renal cell carcinoma (RCC) in von Hippel-Lindau (VHL) disease: Clinical outcome and patterns of radiological response. Fam Cancer 2015;14:309-16. http://dx.doi.org/10.1007/s10689-014-9771-y

Correspondence: Dr. Dong Soo Park, Department of Urology, CHA Bundang Medical Center, CHA University, Seongnam-si, Gyeonggi-do, Korea; dsparkmd@cha.ac.kr 